IFIC $/ 04-63$

\title{
Charge Breaking Minima in the Broken R-parity Minimal Supersymmetric Standard Model
}

\author{
M. Hirsch, $1, *$ C. Hugonie,, 1+ J. C. Romão, ${ }^{2,+}$ and J. W. F. Valle ${ }^{1, \S}$ \\ ${ }^{1}$ AHEP Group, Instituto de Fúsica Corpuscular - C.S.I.C./Universitat de València \\ Edificio Institutos de Paterna, Apt 22085, E-46071 Valencia, Spain \\ ${ }^{2}$ Departamento de Física and CFTP, Instituto Superior Técnico \\ Av. Rovisco Pais 1, 1049-001 Lisboa, Portugal
}

\begin{abstract}
We reconsider the possible presence of charge and colour breaking minima in the scalar potential of the minimal supersymmetric standard model (MSSM) and its minimal generalization with R-parity explicitly broken by bilinear terms (RMSSM). First we generalize some results previously derived for the MSSM case. Next we investigate how robust is the MSSM against its RMSSM extension. We examine the constraints on the RMSSM parameter space that follow from the required absence of charge breaking minima in the scalar potential. We point out the possibility of generating non-zero vacuum expectation values for the charged Higgs field which is not present in the MSSM. However, given the smallness of neutrino masses indicated by neutrino oscillation data, we show that the RMSSM represents only a slight perturbation of the MSSM and is thus as safe (or unsafe) as the MSSM itself from unwanted minima in the scalar potential.
\end{abstract}

PACS numbers: 14.60.Pq, 12.60.Jv, 14.80.Cp

Keywords: supersymmetry; neutrino mass and mixing

*Electronic address: mahirsch@ific.uv.es

${ }^{\dagger}$ Electronic address: hugonie@ific.uv.es

${ }^{\ddagger}$ Electronic address: jorge.romao@ist.utl.pt

§Electronic address: valle@ific.uv.es 


\section{INTRODUCTION}

Softly broken supersymmetric models contain a fairly large number of scalar fields not present in the standard model. Their existence leads to a complicated scalar potential, which might contain undesirable minima which spontaneously break charge and/or color symmetry, a situation which can not happen within the Standard Model. The condition that the "realistic" minimum is the global minimum of the theory can be used to obtain restrictions on the parameter space of supersymmetric models, as already realized more than 20 years ago [1, 2, 3]. This way a disadvantage of supersymmetry may turn into a virtue by shedding some light into the unknown supersymmetry breaking mechanism itself.

Due to the enormous complexity of the full scalar potential in the minimal supersymmetric extension of the standard model (MSSM) early papers on this subject [1, 2, 3, 4] have only analyzed particular, but especially dangerous directions in field-space. Casas et al [5] have presented a more detailed analysis of this subject. They were able to show that in the constrained MSSM (CMSSM) with minimal supergravity boundary conditions strong constraints arise ruling out sizeable parts of the parameter space [5].

Similar studies in R-parity violating versions of the MSSM, however, have not been published ${ }^{1}$. Our main goal is to present a detailed analysis of the 'unboundedfrom-below' (UFB) as well as charge/colour breaking minima (CCB) in the bilinear R-parity breaking model (RMSSM) 7]. This model breaks lepton number and $\mathrm{R}$-parity explicitly through the simplest bilinear terms. The justification for such emphasis is threefold.

First, it represents the simplest possible scheme of $\mathrm{R}$-parity violation, a mere six parameter extension of the MSSM. It is therefore interesting to investigate the "stability" of the MSSM against such "innocuous" perturbation. For this reason we can also call this model the generalized MSSM where R-parity breaks in the minimal way. Second, this model is motivated by the fact that it produces the paradigm for the idea that supersymmetry is the origin of neutrino mass [8], leading to a pattern of neutrino masses [9] that successfully describes current neutrino data [10]. Last, but not least, it represents the only model of $\mathrm{R}$-parity breaking consistent

\footnotetext{
${ }^{1}$ The work of Abel and Savoy [6] contains a discussion on the possibility of lifting flat directions by adding explicit trilinear $\mathrm{R}$-parity violating terms to the superpotential. However, they discuss the impact of bilinear terms only briefly. This is our main emphasis.
} 
with a spontaneous violation of $\mathrm{R}$-parity 11, 12], where it is the vacuum, not the fundamental theory, that breaks the symmetry.

In this model the atmospheric neutrino mass scale [13] is generated at the treelevel, through the mixing of the three neutrinos with the neutralinos 14], in an effective "low-scale" variant of the seesaw mechanism. In contrast, the solar mass and mixings needed to account for solar neutrino data [15, 16] are generated radiatively [9].

A very important difference between such a supersymmetric approach to the origin of neutrino mass and seesaw-type schemes, is that here the dimension-five operator responsible for (Majorana) neutrino masses is generated at an accessibly low energy scale - namely the weak scale. This makes this model potentially testable by experiment. In fact it has been shown that such a low-scale scheme for neutrino masses has the advantage of being testable also "outside" the realm of neutrino physics experiments. Although neutrino properties can not be predicted from first principles, interpreting current neutrino data in this framework implies unambiguous tests of the theory at accelerator experiments [17, 18, 19, 20, 21] which can potentially be used to falsify the model.

This paper is organized as follows. In the next section we will briefly recall some basics of the discussion on CCB and UFB bounds in the MSSM. This will serve as a basis for section 3, where we will discuss new features related to the $\mathrm{R}$-parity violating terms. We show how the bounds from unbounded-from-below directions have to be modified, once non-zero bilinear $\mathrm{R}$-parity violating (BRpV) terms are allowed. We point out the novel possibility to generate a non-zero vacuum expectation value of the charged Higgs field, albeit in regions of parameter space which are now excluded by neutrino physics [10]. We show that, given current data on neutrino masses, bilinear R-parity violation can be understood as a small perturbation of the MSSM. From the point of view of charge breaking minima the RMSSM is thus as safe (or unsafe) as the MSSM itself. We will then close with a short summary.

\section{REVIEW OF THE MSSM RESULTS ON UFB AND CCB}

To set up the notation, the superpotential of the MSSM can be written as

$$
W=\varepsilon_{a b}\left[h_{U}^{i j} \widehat{Q}_{i}^{a} \widehat{U}_{j} \widehat{H}_{u}^{b}+h_{D}^{i j} \widehat{Q}_{i}^{b} \widehat{D}_{j} \widehat{H}_{d}^{a}+h_{E}^{i j} \widehat{L}_{i}^{b} \widehat{R}_{j} \widehat{H}_{d}^{a}-\mu \widehat{H}_{d}^{a} \widehat{H}_{u}^{b}\right]
$$


Here, $h_{U}^{i j}, h_{D}^{i j}$ and $h_{E}^{i j}$ are $3 \times 3$ Yukawa matrices, $\widehat{Q}, \widehat{U}$ and $\widehat{D}$ are quark doublet and singlet superfields and $\widehat{L}$ and $\widehat{R}$ are the usual lepton doublet and singlet fields. Supersymmetry must be broken and the most general set of soft breaking terms allowed by the standard model gauge group under the assumption of lepton number conservation can be written as

$$
\begin{gathered}
V_{S B}=M_{Q}^{i j 2} \widetilde{Q}_{i}^{a *} \widetilde{Q}_{j}^{a}+M_{U}^{i j 2} \widetilde{U}_{i} \widetilde{U}_{j}^{*}+M_{D}^{i j 2} \widetilde{D}_{i} \widetilde{D}_{j}^{*}+M_{L}^{i j 2} \widetilde{L}_{i}^{a *} \widetilde{L}_{j}^{a}+M_{R}^{i j 2} \widetilde{R}_{i} \widetilde{R}_{j}^{*}+\sum_{i=1}^{2} m_{H_{i}}^{2} H_{i}^{a *} H_{i}^{a} \\
+\left[-\frac{1}{2} \sum_{i=1}^{3} M_{i} \lambda_{i} \lambda_{i}+\varepsilon_{a b}\left(A_{U}^{i j} h_{U}^{i j} \widetilde{Q}_{i}^{a} \widetilde{U}_{j} H_{u}^{b}+A_{D}^{i j} h_{D}^{i j} \widetilde{Q}_{i}^{b} \widetilde{D}_{j} H_{d}^{a}+A_{E}^{i j} h_{E}^{i j} \widetilde{L}_{i}^{b} \widetilde{R}_{j} H_{d}^{a}\right.\right. \\
\left.\left.-B \mu H_{d}^{a} H_{u}^{b}\right)+ \text { h.c. }\right]
\end{gathered}
$$

The Higgs doublets giving mass to the standard model fermions are

$$
H_{d}=\left(\begin{array}{c}
H_{d}^{0} \\
H_{d}^{-}
\end{array}\right), \quad H_{u}=\left(\begin{array}{c}
H_{u}^{+} \\
H_{u}^{0}
\end{array}\right)
$$

and the parameters in Eq. (22) are to be understood at some renormalization scale $Q$ chosen to minimize the effects of the one loop corrections. This way we can neglect in the analysis the effect of the one loop radiative corrections [5]. Without loss of generality, we now consider that the fields take the following vev's ${ }^{2}$,

$$
\left\langle H_{u}^{+}\right\rangle=0, \quad\left\langle H_{d}^{-}\right\rangle=v_{-}, \quad\left\langle H_{d}^{0}\right\rangle=v_{d}, \quad\left\langle H_{u}^{0}\right\rangle=v_{u}
$$

to obtain

$$
\begin{aligned}
V_{\text {Higgs }}= & \left(m_{H_{u}}^{2}+\mu^{2}\right) v_{u}^{2}+\left(m_{H_{d}}^{2}+\mu^{2}\right)\left(v_{d}^{2}+v_{-}^{2}\right)-2 B \mu v_{u} v_{d}-\frac{1}{2} g^{2} v_{u}^{2} v_{d}^{2} \\
& +\frac{1}{8}\left(g^{2}+g^{\prime 2}\right)\left(v_{u}^{4}+v_{d}^{4}+v_{-}^{4}+2 v_{d}^{2} v_{-}^{2}\right)+\frac{1}{4}\left(g^{2}-g^{\prime 2}\right)\left(v_{d}^{2}+v_{-}^{2}\right) v_{u}^{2}
\end{aligned}
$$

This Higgs potential has the property that $v_{-}=0$. To see this we note that the potential can be written in the form,

$$
V_{\text {Higgs }}=C_{4} v_{-}^{4}+C_{2} v_{-}^{2}+C_{0}
$$

where

$$
\begin{aligned}
& C_{4}=\frac{1}{8}\left(g^{2}+g^{\prime 2}\right) \\
& C_{2}=\frac{1}{4}\left(g^{2}-g^{\prime 2}\right) v_{u}^{2}+\frac{1}{4}\left(g^{2}+g^{\prime 2}\right) v_{d}^{2}+\left(m_{H_{d}}^{2}+\mu^{2}\right) \\
& C_{0}=\frac{1}{8}\left(g^{2}+g^{\prime 2}\right)\left(v_{u}^{2}-v_{d}^{2}\right)^{2}+\left(m_{H_{u}}^{2}+\mu^{2}\right) v_{u}^{2}+\left(m_{H_{d}}^{2}+\mu^{2}\right) v_{d}^{2}-2 B \mu v_{u} v_{d}
\end{aligned}
$$

\footnotetext{
${ }^{2}$ Our normalization here for the vev's differs from Refs. [7, 9] by a factor of $\sqrt{2}$.
} 
Now since $g>g^{\prime}$ we must have $C_{2}>0$, unless $m_{H_{d}}^{2}+\mu^{2}<0^{3}$. Therefore the minimum of the Higgs potential occurs for vanishing vev of the charged Higgs boson.

By using the minimization equations,

$$
\begin{aligned}
& 0=-2 B \mu v_{d}+2\left(m_{H_{u}}^{2}+\mu^{2}\right) v_{u}-\frac{1}{2}\left(g^{2}+g^{2}\right)\left(v_{d}^{2}-v_{u}^{2}\right) v_{u} \\
& 0=-2 B \mu v_{u}+2\left(m_{H_{d}}^{2}+\mu^{2}\right) v_{d}+\frac{1}{2}\left(g^{2}+g^{\prime 2}\right)\left(v_{d}^{2}-v_{u}^{2}\right) v_{d}
\end{aligned}
$$

one can find the value of the Higgs potential at the real minimum,

$$
V_{M I N}=-\frac{1}{8}\left(g^{2}+g^{\prime 2}\right)\left(v_{u}^{2}-v_{d}^{2}\right)^{2}
$$

Eq. (9) will be important to compare with the values of other (and potentially deeper) minima.

Before starting the discussion of the dangerous directions, a word of caution should be added, namely, that the condition that the realistic minimum is the global one might actually be too conservative. In fact, it is possible that the universe resides in a false vacuum which is stable because the tunneling time into the global minimum is large with respect to the age of the universe. In this sense, CCB and UFB constraints on the supersymmetric parameter space are sufficient but might not be necessary, see for example [22, 23]. However, we will not follow this line of reasoning any further.

\section{A. UFB directions}

The 'unbounded-from-below' (UFB) directions are those where the quartic Dterms vanish and some coefficient(s) quadratic in the vev's are negative. Then the potential at the weak scale seems to be unbounded from below. However, this is a slight misnomer, since if one assumes that all soft masses are positive at the high unification scale, it appears that these dangerous directions are not really unbounded from below but there exists a true local minimum at some large scale. It then must be checked that this local minimum is not deeper than the physical one. As was shown in Ref. [5] there are three kinds of such directions. The first and most obvious one corresponds to the D-flat direction where $\left|v_{u}\right|=\left|v_{d}\right|$, all other vev's being zero.

\footnotetext{
${ }^{3}$ Casas et al. 5] assume that only $m_{H_{u}}^{2}+\mu^{2}$ can be negative. Even though in mSugra at very large $\tan \beta$ values $m_{H_{d}}^{2}+\mu^{2}<0$ can occur in exceptional cases, we will follow their assumption.
} 
The potential along this direction reads,

$$
V_{U F B-1}=\left(m_{H_{u}}^{2}+m_{H_{d}}^{2}+2 \mu^{2}-2|B \mu|\right) v_{u}^{2}
$$

and a sufficient condition to avoid developping a deep minimum at large values of the field is

$$
m_{H_{u}}^{2}+m_{H_{d}}^{2}+2 \mu^{2}-2|B \mu|>0 .
$$

In principle, one should check the depth of the true minimum along the dangerous direction when this coefficient is negative. For simplicity, we will stick however to the condition given in Eq. (11).

The second dangerous direction corresponds to the case where a slepton $L_{i}$ takes a vev $v_{i}$. Then a combination of $v_{u}, v_{d}$ and $v_{i}$ can cancel the D-term and the potential reads,

$$
V_{U F B-2}=\left(m_{H_{u}}^{2}+\mu^{2}+m_{L_{i}}^{2}-\frac{|B \mu|^{2}}{m_{H_{d}}^{2}+\mu^{2}-m_{L_{i}}^{2}}\right) v_{u}^{2}-\frac{2 m_{L_{i}}^{4}}{g^{2}+g^{\prime 2}}
$$

which constrains the coefficient of the quadratic term as

$$
m_{H_{u}}^{2}+\mu^{2}+m_{L_{i}}^{2}-\frac{|B \mu|^{2}}{m_{H_{d}}^{2}+\mu^{2}-m_{L_{i}}^{2}}>0 .
$$

Note that in the case of a universal $m_{0}$ at the unification scale the $m_{L_{i}}$ are usually the smallest soft masses at the weak scale. Dropping the universality assumption the bound obtained for $m_{L_{i}}$, Eq. (13), must be verified for the squark soft masses as well.

Finally the last UFB direction corresponds to the case where $v_{d}=0$ but we have a neutral slepton $L_{i}$ with nonzero vev, like in the UFB-2 case. This direction is both $\mathrm{D}$ - and F-flat. The difference with respect to UFB-2 is that the F-term is canceled by giving vev's to the charged sleptons. The resulting potential reads

$$
V_{U F B-3}=\left(m_{H_{u}}^{2}+m_{L_{i}}^{2}\right) v_{u}^{2}+\frac{|\mu|}{h_{e_{j}}}\left(m_{L_{i}}^{2}+m_{L_{j}}^{2}+m_{e_{j}}^{2}\right) v_{u}-\frac{2 m_{L_{i}}^{4}}{g^{2}+g^{\prime 2}}
$$

Since $m_{H_{u}}^{2}$ must be negative in order to break electroweak symmetry and $m_{L_{i}}^{2}$ is small when one assumes universality of the soft terms, the coefficient quadratic in $v_{u}$ is generally negative. As shown in Refs. [5, 6] in the case of universal soft masses at the GUT scale, the condition that the minimum along this UFB-3 direction is not deeper than the physical minimum implies $m_{0}>\alpha M_{1 / 2}$, where $\alpha$ is a coefficient of $\mathcal{O}(1)$. 


\section{B. CCB minima}

For the classical CCB minima, dangerous negative contributions to the scalar potential are generated by cubic ( $A$-type) soft supersymmetry breaking terms. Therefore these directions cannot be F-flat, but they are still D-flat. The traditional bound of Ref. [1] corresponds to the case where

$$
\left\langle Q^{1}\right\rangle=\left\langle H_{u}^{2}\right\rangle=\langle U\rangle=v
$$

all other vev's vanishing. This choice cancels the D-term and the potential reads,

$$
V_{C C B}=v^{2}\left(3 h_{u}^{2} v^{2}+2 A_{u} h_{u} v+m_{H_{u}}^{2}+\mu^{2}+m_{Q}^{2}+m_{U}^{2}\right)
$$

In order to avoid a very deep color and charge breaking minimum we must make sure that the parenthesis in Eq. (16) never vanishes, which happens if the corresponding second order equation can not have real solutions. This leads to the well known condition,

$$
\left|A_{u}\right|^{2}<3\left(m_{H_{u}}^{2}+\mu^{2}+m_{Q}^{2}+m_{U}^{2}\right)
$$

A more complete and general analysis of this and similarly dangerous directions can be found in Ref. [5]. Note again, that the bound given in Eq.(17) for $A_{u}$ must be checked for all $A$-terms in the general non-universal MSSM.

\section{UFB AND CCB IN THE RMSSM}

The RMSSM is simply the bilinear R-parity violating model, defined by the following superpotential [7]

$$
W=W_{M S S M}+\varepsilon_{a b} \epsilon_{i} \widehat{L}_{i}^{a} \widehat{H}_{u}^{b}
$$

and corresponding soft supersymmetry breaking terms,

$$
V_{S B}=V_{M S S M}+B_{i} \epsilon_{i} \widetilde{L}_{i}^{a} H_{u}^{b}
$$

It is therefore a rather mild extension of the MSSM. In the following it will be sufficient to consider for simplicity only a one generation version of the model ${ }^{4}$.

\footnotetext{
${ }^{4}$ We do not believe that this simplification has any impact on the following discussion, since neutrino oscillation data require $\frac{\epsilon}{\mu} \ll 1$ and intergenerational effects between different families of leptons due to $\mathrm{BRpV}$ terms scale as $\left(\frac{\epsilon}{\mu}\right)^{2}$.
} 
We are mainly interested in studying how the appearance of the new terms in the superpotential (and in $V_{S B}$ ) changes the conclusions which hold for the MSSM. Since the MSSM is the limit of the RMSSM when $\epsilon \rightarrow 0$ we expect that the results of the MSSM will hold in that limit. Note also that the structure of the trilinear terms is not modified, so conclusions like those of Eq. (17) are expected also to hold in our case. Defining

$$
\left\langle H_{u}^{+}\right\rangle=0, \quad\left\langle H_{d}^{-}\right\rangle=v_{-}, \quad\left\langle H_{d}^{0}\right\rangle=v_{d}, \quad\left\langle H_{u}^{0}\right\rangle=v_{u}, \quad\left\langle L^{0}\right\rangle=v^{\prime}, \quad\left\langle L^{-}\right\rangle=v_{-}^{\prime}
$$

one finds for the scalar potential

$$
\begin{aligned}
V= & M_{H_{u}}^{2} v_{u}^{2}+M_{H_{d}}^{2}\left(v_{d}^{2}+v_{-}^{2}\right)+M_{L}^{2}\left(v^{\prime 2}+v_{-}^{\prime 2}\right)-2 B \mu v_{d} v_{u}+2 B^{\prime} \epsilon v_{u} v^{\prime} \\
& +\epsilon^{2}\left(v_{u}^{2}+v_{-}^{\prime 2}+v^{\prime 2}\right)+\mu^{2}\left(v_{u}^{2}+v_{d}^{2}+v_{-}^{2}\right)-2 \mu \epsilon\left(v^{\prime} v_{d}+v_{-} v_{-}^{\prime}\right) \\
& +\frac{g^{2}}{8}\left[\left(v_{u}^{2}-v_{d}^{2}-v^{\prime 2}+v_{-}^{2}+v_{-}^{\prime 2}\right)^{2}+4\left(v_{d} v_{-}+v^{\prime} v_{-}^{\prime}\right)^{2}\right] \\
& +\frac{g^{\prime 2}}{8}\left(v_{u}^{2}-v_{d}^{2}-v^{\prime 2}-v_{-}^{2}-v_{-}^{\prime 2}\right)^{2}
\end{aligned}
$$

where $B^{\prime}$ characterizes the soft supersymmetry and R-parity violating bilinear term. We note that it is not possible to have an UFB direction with non vanishing charged vev's in this potential, because the $\mathrm{D}$-terms can not be made to vanish for $v_{-}$and $v_{-}^{\prime}$ different from zero. The minimization equations can be found in the usual way taking derivatives with respects to the fields

$$
\begin{aligned}
0= & {\left[2\left(M_{H_{d}}^{2}+\mu^{2}\right)-\frac{g^{2}}{2}\left(v_{u}^{2}-v_{d}^{2}-v^{\prime 2}-v_{-}^{2}+v_{-}^{\prime 2}\right)-\frac{g^{\prime 2}}{2}\left(v_{u}^{2}-v_{d}^{2}-v^{\prime 2}-v_{-}^{2}-v_{-}^{\prime 2}\right)\right] v_{d} } \\
& -\left(2 \epsilon \mu-g^{2} v_{-} v_{-}^{\prime}\right) v^{\prime}-2 B \mu v_{u} \\
0= & {\left[\frac{g^{2}}{2}\left(v_{u}^{2}-v_{d}^{2}-v^{\prime 2}+v_{-}^{2}+v_{-}^{\prime 2}\right)+\frac{g^{\prime 2}}{2}\left(v_{u}^{2}-v_{d}^{2}+v^{\prime 2}-v_{-}^{2}-v_{-}^{\prime 2}\right)\right] v_{u} } \\
& +2\left(M_{H_{u}}^{2}+\mu^{2}+\epsilon^{2}\right) v_{u}+2\left(B^{\prime} \epsilon v^{\prime}-B \mu v_{d}\right) \\
0 & {\left[2\left(M_{L}^{2}+\epsilon^{2}\right)-\frac{g^{2}}{2}\left(v_{u}^{2}-v_{d}^{2}-v^{\prime 2}+v_{-}^{2}-v_{-}^{\prime 2}\right)-\frac{g^{\prime 2}}{2}\left(v_{u}^{2}-v_{d}^{2}-v^{\prime 2}-v_{-}^{2}-v_{-}^{\prime 2}\right)\right] v^{\prime} } \\
& -\left(2 \epsilon \mu-g^{2} v_{-} v_{-}^{\prime}\right) v_{d}+2 B^{\prime} \epsilon v_{u} \\
0 & {\left[2\left(M_{H_{d}}^{2}+\mu^{2}\right)+\frac{g^{2}}{2}\left(v_{u}^{2}+v_{d}^{2}-v^{\prime 2}+v_{-}^{2}+v_{-}^{\prime 2}\right)-\frac{g^{\prime 2}}{2}\left(v_{u}^{2}-v_{d}^{2}-v^{\prime 2}-v_{-}^{2}-v_{-}^{\prime 2}\right)\right] v_{-} } \\
& -\left(2 \epsilon \mu-g^{2} v_{d} v^{\prime}\right) v_{-}^{\prime} \\
0 & {\left[2\left(M_{L}^{2}+\epsilon^{2}\right)+\frac{g^{2}}{2}\left(v_{u}^{2}-v_{d}^{2}+v^{\prime 2}+v_{-}^{2}+v_{-}^{\prime 2}\right)-\frac{g^{\prime 2}}{2}\left(v_{u}^{2}-v_{d}^{2}-v^{\prime 2}-v_{-}^{2}-v_{-}^{\prime 2}\right)\right] v_{-}^{\prime} } \\
& \quad\left(2 \epsilon \mu-g^{2} v_{d} v^{\prime}\right) v_{-}
\end{aligned}
$$


Since we are dealing with a set of five coupled equations this system is difficult to solve for the vev's. We can however use the following trick. Instead of solving for the five vev's we try to solve those equations for the three soft masses squared $M_{H_{u}}^{2}$, $M_{H_{d}}^{2}$ and $M_{L}^{2}$ [12] and for the charged vev's. Using this approach we could find two types of solutions.

Before discussing the general case, however, we consider first the limit in which RMSSM is considered a perturbation of the MSSM. This is a reasonable approach since the BRpV parameters must be small to account for the neutrino data 9$]$. Therefore we can pose the following question. Suppose that in the limit $\epsilon \rightarrow 0$ the parameters are such that the MSSM has no UFB directions or CCB minima. This means $v_{u} \neq 0, v_{d} \neq 0$ and $v^{\prime}=v_{-}=v_{-}^{\prime}=0$. If we now consider a small non-vanishing value for the $\epsilon$ what will be the corresponding minimum? In order to answer this question in perturbation theory we write

$$
v_{d}=\sum_{i=0}^{\infty} v_{d}^{(i)} \epsilon^{i}, v_{u}=\sum_{i=0}^{\infty} v_{u}^{(i)} \epsilon^{i}, v^{\prime}=\sum_{i=0}^{\infty} v^{\prime(i)} \epsilon^{i}, v_{-}=\sum_{i=0}^{\infty} v_{-}^{(i)} \epsilon^{i}, v_{-}^{\prime}=\sum_{i=0}^{\infty} v_{-}^{\prime(i)} \epsilon^{i}
$$

Now we substitute back in the extremum Eq. (22) and solve order by order in perturbation theory. The result that we get is as follows,

$$
\begin{aligned}
& v_{d}=v_{d}^{(0)}+v_{d}^{(2)} \epsilon^{2}+v_{d}^{(4)} \epsilon^{4}+\cdots \\
& v_{u}=v_{u}^{(0)}+v_{u}^{(2)} \epsilon^{2}+v_{u}^{(4)} \epsilon^{4}+\cdots \\
& v^{\prime}=v^{\prime(1)} \epsilon+v^{\prime(3)} \epsilon^{3}+v^{\prime(5)} \epsilon^{5}+\cdots \\
& v_{-}=0 \\
& v_{-}^{\prime}=0
\end{aligned}
$$

where $v_{u}^{(0)}, v_{d}^{(0)}$ are the MSSM values for $\epsilon=0$. This is precisely the solution of type I that we will discuss shortly. Note that if $\epsilon \neq 0$ then also $v^{\prime} \neq 0$. In fact,

$$
v^{\prime}=\frac{\mu v_{d}^{(0)}-B^{\prime} v_{u}^{(0)}}{M_{L}^{2}-\frac{1}{4}\left(g^{2}+g^{\prime 2}\right)\left(v_{u}^{(0)}{ }^{2}-v_{d}^{(0)_{2}}\right)} \epsilon+\cdots
$$

So we can formulate the following important result: If we start with the MSSM parameters such that in the limit $\epsilon \rightarrow 0$ the minimum has no UFB or CCB problems, then by turning on perturbatively a small value for $\epsilon$ we get a correspondingly safe minimum of the RMSSM. However, as we will now discuss, in general there are two types of solutions for the minimum equations. 


\section{Type $I$}

This solution corresponds to the case where the charged vev's vanish. We are then in the situation studied usually [7] in the bilinear R-parity model. We get

$$
\begin{aligned}
M_{H_{d}}^{2} & =\epsilon \mu \frac{v^{\prime}}{v_{d}}-\mu^{2}+B \mu \frac{v_{u}}{v_{d}}+\frac{g^{2}+g^{\prime 2}}{4}\left(v_{u}^{2}-v^{\prime 2}-v_{d}^{2}\right) \\
M_{H_{u}}^{2} & =-\epsilon^{2}-\mu^{2}+B \mu \frac{v_{d}}{v_{u}}-B^{\prime} \epsilon \frac{v^{\prime}}{v_{u}}-\frac{g^{2}+g^{\prime 2}}{4}\left(v_{u}^{2}-v^{\prime 2}-v_{d}^{2}\right) \\
M_{L}^{2} & =-\epsilon^{2}+\epsilon\left(\mu \frac{v_{d}}{v^{\prime}}-B^{\prime} \frac{v_{u}}{v^{\prime}}\right)+\frac{g^{2}+g^{\prime 2}}{4}\left(v_{u}^{2}-v^{\prime 2}-v_{d}^{2}\right) \\
v_{-} & =0 \\
v_{-}^{\prime} & =0
\end{aligned}
$$

This corresponds to the neutral Higgs potential that we will discuss further below. Here we just note that the value of the potential at the minimum can be shown to be

$$
V_{B R p V}=-\frac{g^{2}+g^{\prime 2}}{8}\left(v_{u}^{2}-v_{d}^{2}-v^{\prime 2}\right)^{2}
$$

Type II

In the general case we can find the solutions of the minimization equations in the following way. We start by solving the first three equations in Eq. (22) for the soft masses. We get,

$$
\begin{aligned}
M_{H_{d}}^{2} & =M_{H_{d}}^{2}(0)-\frac{1}{4}\left(g^{2}-g^{\prime 2}\right)\left(v_{-}^{2}+v_{-}^{\prime 2}\right) \\
M_{H_{u}}^{2} & =M_{H_{u}}^{2}(0)-\frac{1}{4}\left(g^{2}+g^{\prime 2}\right)\left(v_{-}^{2}+v_{-}^{\prime 2}\right)-\frac{1}{2} g^{2} \frac{v^{\prime}}{v_{d}} v_{-}^{\prime} v_{-} \\
M_{L}^{2} & =M_{L}^{2}(0)+\frac{1}{4}\left(g^{2}-g^{\prime 2}\right) v_{-}^{2}-\frac{1}{4}\left(g^{2}+g^{\prime 2}\right) v_{-}^{\prime 2}-\frac{1}{2} g^{2} \frac{v^{\prime}}{v_{d}} v_{-}^{\prime} v_{-}
\end{aligned}
$$

where $M_{H_{d}}^{2}(0), M_{H_{u}}^{2}(0)$ and $M_{L}^{2}(0)$ are the soft masses when $v_{-}=v_{-}^{\prime}=0$ and are given in Eq. (26). Now we substitute Eq. (28) into the last two equations in Eq. (22) to obtain,

$$
\begin{aligned}
& 0=-g^{2}\left(v^{\prime 2} v_{-}-v^{\prime} v_{d} v_{-}^{\prime}+v_{-}^{2} v_{-}^{\prime} \frac{v^{\prime}}{v_{d}}-v_{-} v_{-}^{\prime 2}\right)+2 \epsilon \mu\left(v_{-} \frac{v^{\prime}}{v_{d}}-v_{-}^{\prime}\right)+2 B \mu v_{-} \frac{v_{u}}{v_{d}}+g^{2} v_{-} v_{u}^{2} \\
& 0=g^{2}\left(v^{\prime 2} v_{-}-v^{\prime} v_{d} v_{-}^{\prime}+v_{-}^{2} v_{-}^{\prime} \frac{v^{\prime}}{v_{d}}-v_{-} v_{-}^{\prime 2}\right) \frac{v_{d}}{v^{\prime}}-2 \epsilon \mu\left(v_{-} \frac{v^{\prime}}{v_{d}}-v_{-}^{\prime}\right) \frac{v_{d}}{v^{\prime}}
\end{aligned}
$$




$$
-2 B^{\prime} \epsilon v_{-}^{\prime} \frac{v_{u}}{v^{\prime}}+g^{2} v_{-}^{\prime} v_{u}^{2}
$$

Multiplying the second of the equations in Eq. (29) by $v^{\prime} / v_{d}$ and adding them one obtains,

$$
v_{-}^{\prime}=\kappa v_{-}
$$

where

$$
\kappa=\frac{2 B \mu+g^{2} v_{d} v_{u}}{2 B^{\prime} \epsilon-g^{2} v^{\prime} v_{u}}
$$

Finally we use Eq. (30) to reduce either one of Eq. (29) to

$$
0=v_{-}\left(D_{2} v_{-}^{2}-D_{0}\right)
$$

where

$$
\begin{aligned}
& D_{2}=g^{2}\left(\kappa^{2}-\frac{v^{\prime}}{v_{d}} \kappa\right) \\
& D_{0}=g^{2}\left(v^{\prime 2}-v_{d} v^{\prime} \kappa-v_{u}^{2}\right)-\left(B v_{u}+\epsilon v^{\prime}\right) \frac{2 \mu}{v_{d}}+2 \epsilon \mu \kappa
\end{aligned}
$$

Eq. (32) has the trivial solution $v_{-}=0$ which corresponds to type $\mathbf{I}$, the BRpV solutions. However, if

$$
\frac{D_{0}}{D_{2}}>0
$$

we have a new type of solutions for the minimization equations,

$$
v_{-}= \pm \sqrt{\frac{D_{0}}{D_{2}}}, \quad v_{-}^{\prime}=\kappa v_{-}
$$

As $D_{0,2}$ do not have in general a well defined sign it can happen that such solutions do exist for some combination of the parameters. We will discuss this later in more detail.

\section{A. UFB Directions}

We have seen before that for the Higgs potential of the RMSSM the UFB directions can only arise when the charged Higgs vev's vanish, otherwise it is not possible to cancel the quartic D-terms. The neutral Higgs potential obtained from Eq. (21) when $v_{-}=0, v_{-}^{\prime}=0$ is given by

$$
\begin{aligned}
V_{\text {Neutral }}= & \left(M_{H_{u}}^{2}+\epsilon^{2}+\mu^{2}\right) v_{u}^{2}+\left(M_{H_{d}}^{2}+\mu^{2}\right) v_{d}^{2}+\left(M_{L}^{2}+\epsilon^{2}\right) v^{\prime 2} \\
& -2 B \mu v_{d} v_{u}+2 B^{\prime} \epsilon v_{u} v^{\prime}-2 \mu \epsilon v^{\prime} v_{d}+\frac{g^{2}+g^{\prime 2}}{8}\left(v_{u}^{2}-v_{d}^{2}-v^{\prime 2}\right)^{2}
\end{aligned}
$$


From this equation we can see that we can make the D-term vanish if we choose the condition

$$
v_{u}^{2}=v_{d}^{2}+v^{\prime 2}
$$

To implement this condition it is convenient to write

$$
v_{d}=v_{u} \cos \theta, \quad v^{\prime}=v_{u} \sin \theta
$$

Then we get

$$
V_{\text {Neutral }}=B(\theta) v_{u}^{2}
$$

where

$$
\begin{aligned}
B(\theta)=[ & M_{H_{u}}^{2}+\epsilon^{2}+\mu^{2}+\left(M_{H_{d}}^{2}+\mu^{2}\right) \cos ^{2} \theta+\left(M_{L}^{2}+\epsilon^{2}\right) \sin ^{2} \theta \\
& \left.-2 B \mu \cos \theta+2 B^{\prime} \epsilon \sin \theta-2 \mu \epsilon \sin \theta \cos \theta\right]
\end{aligned}
$$

Therefore the condition for avoiding an UFB direction is that,

$$
B\left(\theta_{\min }\right)>0
$$

where $\theta_{\min }$ is the value of $\theta$ that corresponds to the minimum of $B(\theta)$. Now consider Eq. (40) in the limit $\epsilon \rightarrow 0$ and take the derivative,

$$
\frac{d B}{d \theta}=2 \sin \theta\left[-\left(M_{H_{d}}^{2}+\mu^{2}-M_{L}^{2}\right) \cos \theta+B \mu\right]
$$

The right hand side vanishes when $\theta=0$ and when $\cos \theta=\frac{B \mu}{M_{H_{d}}^{2}+\mu^{2}-M_{L}^{2}}$. These two solutions correspond to the UFB-1 and UFB-2 directions given in Eqs. (11) and (13), respectively, when $\epsilon=0$.

For $\epsilon \neq 0$ it does not seem possible to have an analytical expression for $\theta_{\min }$. However for a given set of parameters it is always easy to verify whether Eq. (41) holds for $\theta \in[0,2 \pi]$. It is also clear from Eq. (40) that the MSSM condition, Eq. (11), is not enough to ensure that we are free from UFB directions. This fact can be best illustrated from figure (11) that shows a typical example.

One can see clearly that starting from a large value of $B(0)$ is not enough to decide upon the sign of $B\left(\theta_{\min }\right)$. However it is easy to check numerically whether $B\left(\theta_{\min }\right)>0$ or not. Therefore, although we lack a simple analytical formula, the criterium for avoiding UFB directions is easily implemented.

Finally we comment briefly on the direction UFB-3. It can be easily shown that at large values of the field the potential in direction UFB-3 is given as

$$
V_{U F B-3}=\left(m_{H_{u}}^{2}+m_{L_{i}}^{2}+\epsilon B^{\prime}\right) v_{u}^{2}+\cdots
$$



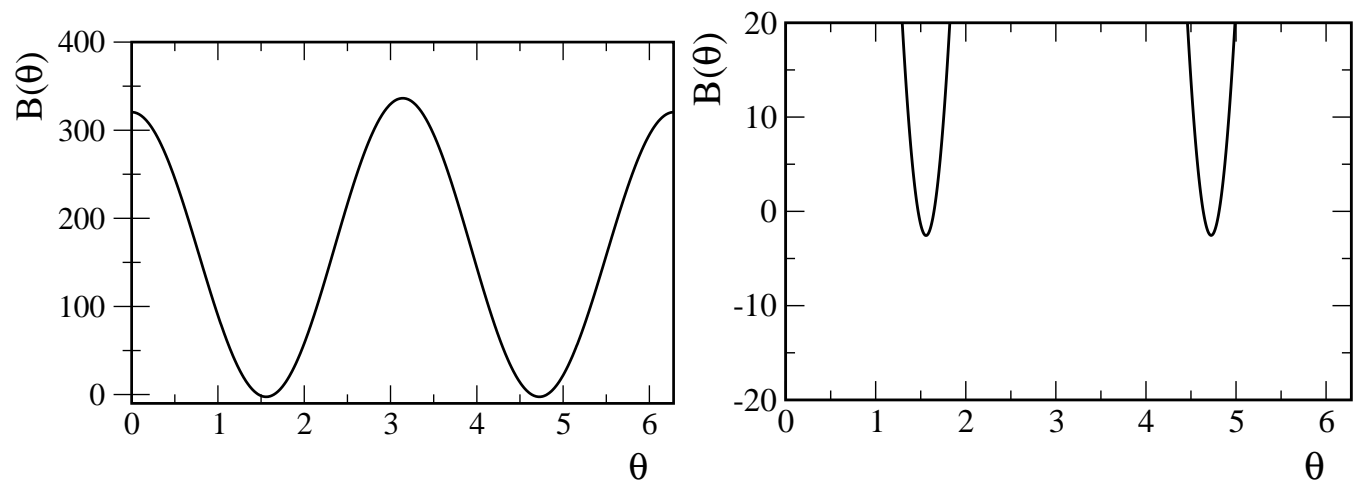

FIG. 1: $B(\theta)$ as a function of $\theta$ for an example where $B\left(\theta_{\min }\right)<0$ but $B(0)>0$. The right panel is an enlarged view of the left one close to the zeros of $B(\theta)$.

where the dots stand for irrelevant terms. Since in our notation $\epsilon B^{\prime}<0$ this leads, in principle, to a slightly more stringent requirement than the one corresponding to the R-parity conserving MSSM. However, since $\frac{\epsilon}{\mu} \sim \mathcal{O}\left(10^{-(3-4)}\right)$ is required by neutrino oscillation data 9], this modification is numerically irrelevant. This is in agreement with the argument presented in Ref. [6].

\section{B. Nonzero charged Higgs and Slepton Vev's}

We now turn to the solutions of type II. We have already seen in Eqs. (32) - (35) that there are potentially dangerous solutions for the Higgs potential with nonzero vev's for the charged scalars. These solutions, if they exist, would provide new CCB solutions different from those already present in the MSSM, as explained above. As can be seen from Eq. (34) such solutions can exist if the parameters satisfy the relation $D_{0} / D_{2}>0$, where the $D_{i}$ are given in Eq. (33).

Since it does not seem possible to give a strict analytic criterion which relates the condition $D_{0} / D_{2}<0$ (guaranteeing the absence of unwanted minima) to the parameters of the potential we have resorted to a numerical scan of the parameter space. Our approach to find the minima of the potential was as follows. We always started with a random set of parameters with zero charged vev's and subject to the requirement that,

$$
v_{u}^{2}+v_{d}^{2}+v^{\prime 2}=v^{2}=\left(2 \sqrt{2} G_{F}\right)^{-1 / 2}=174.1 \mathrm{GeV}
$$

Note that with this procedure we should always have,

$$
|\eta|=\frac{\left|v^{\prime}\right|}{v}<1
$$


We then search for the global minimum numerically. If we find a minimum deeper than the realistic minimum but which breaks charge this part of parameter space should be discarded. Two examples are shown in Fig. (2).
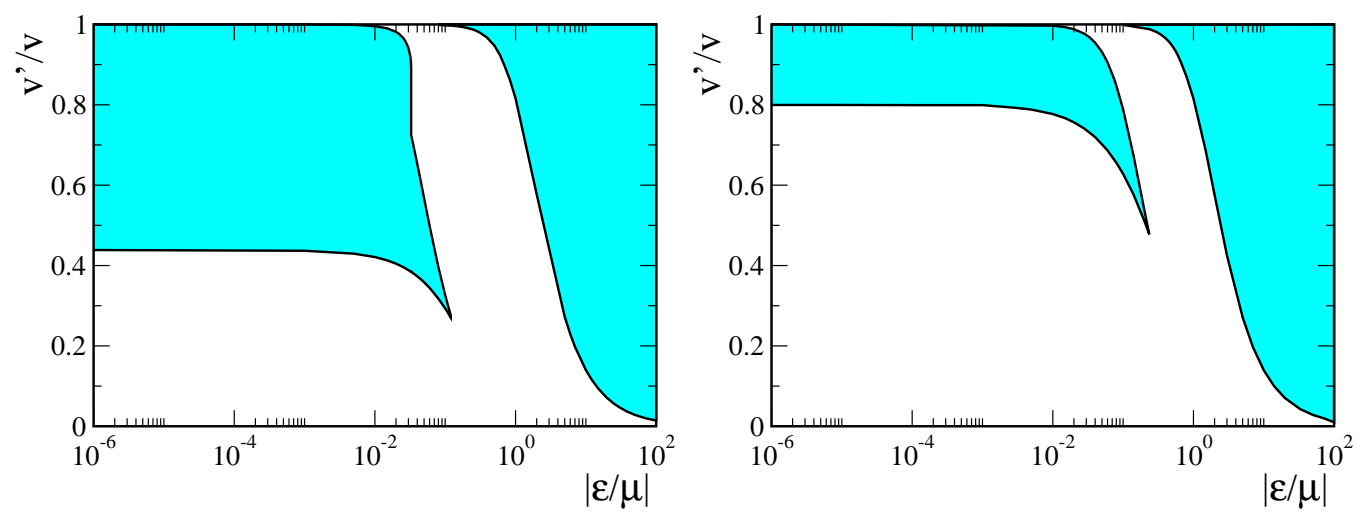

FIG. 2: Range of RMSSM parameters where nonzero charged vev's for the Higgs and slepton fields are favoured over the realistic minimum for two examples of $\tan \beta$, left $\tan \beta=1.05$, right $\tan \beta=1.2$. Here we fix for convenience $B=B^{\prime}=\mu=100 \mathrm{GeV}$. For a discussion see text.

The results shown in Fig. (2) can be understood qualitatively as follows. Starting with the definitions Eqs (31) and (33) and taking into account the smallness of $\frac{\epsilon}{\mu}$ one can show that in the limit $\epsilon \rightarrow 0$ we always have $D_{2}>0$. On the other hand the condition $D_{0}>0$ requires

$$
v^{\prime 2}>v^{2} \frac{\tan ^{2} \beta-1}{1+\tan ^{2} \beta}+\frac{2 B \mu}{g} \frac{\tan ^{2} \beta-1}{\tan \beta} .
$$

Note that this condition is not strictly valid for $\tan \beta \equiv 1$, because in this limit we can no longer neglect the terms proportional to $\epsilon$ in the definitions of $D_{0}$ and $D_{2}$. Eq. (46) shows that charge breaking minima in the limit of small values of $\epsilon$ require that $v^{\prime}$ take up a sizeable fraction of $v$. This trend is clearly visible from Fig. (2). The figure also illustrates how these solutions disappear very quickly with $\tan \beta$ greater than 1.

Although we find it amusing that such solutions exist, we wish to stress that consistency with neutrino data requires $\frac{\epsilon}{\mu} \sim \mathcal{O}\left(10^{-(3-4)}\right)$ and $\frac{v^{\prime}}{v} \sim \mathcal{O}\left(10^{-(3-4)}\right)$. We therefore conclude that the RMSSM is automatically safe from these unwanted minima in those "physical" parts of parameter space which account for the neutrino oscillation data. 


\section{CONCLUSIONS}

We have studied charge breaking minima and unbounded from below directions within bilinear R-parity breaking supersymmetry. Such a "reference model" is nothing but the simplest broken R-parity version of the Minimal Supersymmetric Standard Model. We have first generalized some results obtained previously in the R-parity conserving MSSM. Subsequently we discussed new ways to generate a nonzero vacuum expectation value of the charged Higgs and slepton fields. However, such unwanted solutions occur only in regions of parameter space which are now excluded by neutrino oscillation data.

In summary it can be said that, given the data on neutrino masses, bilinear $\mathrm{R}-$ parity violation can be understood as a small perturbation of the MSSM. From the point of view of CCB and UFB directions the RMSSM is as robust as the R-parityconserving MSSM: it is equally safe from unwanted minima in the same portions of parameter space.

\section{ACKNOWLEDGMENTS}

This work was supported by Spanish grant BFM2002-00345, by the European Commission Human Potential Program RTN network HPRN-CT-2000-00148 and by the European Science Foundation network grant N.86. M.H. is supported by a MCyT Ramon y Cajal contract. JCR was supported by the Portuguese Fundação para a Ciência e a Tecnologia under the contract CFIF-Plurianual and grant POCTI/FNU/4989/2002. We thank Werner Porod for useful discussions.

[1] J. M. Frere, D. R. T. Jones and S. Raby, Nucl. Phys. B 222 (1983) 11.

[2] M. Claudson, L. J. Hall and I. Hinchliffe, Nucl. Phys. B 228 (1983) 501.

[3] H. P. Nilles, M. Srednicki and D. Wyler, Phys. Lett. B 124 (1983) 337.

[4] H. Komatsu, Phys. Lett. B 215 (1988) 323.

[5] J. A. Casas, hep-ph/9707475 J. A. Casas, A. Lleyda and C. Munoz, Phys. Lett. B 389 (1996) 305 hep-ph/9606212; J. A. Casas, A. Lleyda and C. Munoz, Nucl. Phys. B 471 (1996) 3 hep-ph/9507294.

[6] S. A. Abel and C. A. Savoy, Nucl. Phys. B 532 (1998) 3 hep-ph/9803218. 
[7] M. A. Diaz, J. C. Romão and J. W. F. Valle, Nucl. Phys. B 524, 23 (1998) hep-ph/9706315.

[8] For a recent review see M. Hirsch and J. W. F. Valle, New J. Phys. 6 (2004) 76 hep-ph/0405015 and references therein.

[9] M. Hirsch et al., Phys. Rev. D62, 113008 (2000), hep-ph/0004115], Err-ibid. D65 119901 (2002).

[10] M. Maltoni et al, New J. Phys. 6 (2004) 122 hep-ph/0405172. Based on previous paper published in Phys. Rev. D68, 113010 (2003), hep-ph/0309130, which also contain an extensive list of references.

[11] A. Masiero and J. W. F. Valle, Phys. Lett. B 251, 273 (1990).

[12] J. C. Romão, C. A. Santos and J. W. F. Valle, Phys. Lett. B 288, 311 (1992); J. C. Romão, A. Ioannisian and J. W. F. Valle, Phys. Rev. D 55, 427 (1997) hep-ph/9607401 M. Hirsch et al, Phys. Rev. 70, 073012 (2004) hep-ph/0407269.

[13] Y. Fukuda et al. [Super-Kamiokande Collaboration], Phys. Rev. Lett. 81, 1562 (1998); for an update see the talk by E. Kearns at 21st International Conference on Neutrino Physics and Astrophysics (Neutrino 2004) http://neutrino2004.in2p3.fr/

[14] J. R. Ellis et al, Phys. Lett. B 150 (1985) 142; A. Santamaria and J. W. F. Valle, Phys. Lett. B 195 (1987) 423.

[15] Q. R. Ahmad et al. [SNO Collaboration], Phys. Rev. Lett. 89 (2002) 011301 nucl-ex/0204008; Phys. Rev. Lett. 89, 011302 (2002) nucl-ex/0204009.

[16] K. Eguchi et al. [KamLAND Collaboration], Phys. Rev. Lett. 90 (2003) 021802 hep-ex/0212021. For an update see talk by G. Gratta at 21st International Conference on Neutrino Physics and Astrophysics (Neutrino 2004) http://neutrino2004.in2p3.fr/

[17] B. Mukhopadhyaya, S. Roy and F. Vissani, Phys. Lett. B 443 (1998) 191 hep-ph/9808265; A. Datta, B. Mukhopadhyaya and F. Vissani, Phys. Lett. B 492 (2000) 324 hep-ph/9910296.

[18] W. Porod et al, Phys. Rev. D 63 (2001) 115004 hep-ph/0011248.

[19] M. Hirsch et al, Phys. Rev. D 66 (2002) 095006 hep-ph/0207334.

[20] E. J. Chun, D. W. Jung, S. K. Kang and J. D. Park, Phys. Rev. D 66 (2002) 073003 hep-ph/0206030.

[21] M. Hirsch and W. Porod, Phys. Rev. D 68 (2003) 115007 hep-ph/0307364.

[22] A. Kusenko, P. Langacker and G. Segre, Phys. Rev. D 54 (1996) 5824 hep-ph/9602414. 
[23] A. Kusenko and P. Langacker, Phys. Lett. B 391 (1997) 29 hep-ph/9608340. 